\title{
Improvement of biohydrogen production in microbial electrolysis cell (MEC) system by tackling methanogenesis
}

\author{
Matthew Hardhi ${ }^{1}$, Putty Ekadewi ${ }^{1}$, Rita Arbianti ${ }^{1}$, Tania Surya Utami $^{1 *}$, and Heri Hermansyah ${ }^{1}$ \\ ${ }^{1}$ Department of Chemical Engineering, Faculty of Engineering Universitas Indonesia, Depok 16424, Indonesia
}

\begin{abstract}
The increasingly adverse effects of climate change caused by a variety of fossil-based fuel demands an alternative to such fuel. Hydrogen is one of the potential renewable fuel that offers numerous advantages compared to its competitors. However, the dominant hydrogen production methods are still energy-heavy and dependent on fossil-based resources. Microbial electrolysis cell or MEC system is one of the leading solution towards replacing conventional hydrogen production method. A persistent downside to this system in the presence of methanogens that consumes the hydrogen product. This research proposes alternative biological method to control the methanogen colony by introducing isolates of denitrifying bacteria to the system which will act as inhibitor to hydrogenotrophic methanogen. The reactor implemented is a single-chambered, membrane-less $20-\mathrm{ml}$ reactor. Net hydrogen yield produced in the cathodic headspace will be analyzed by gas chromatography (GC). Hydrogen yield for reactor with enriched cathode is expected to be higher in comparison to unenriched reactor, as nitrogen oxides produced during the metabolism of the denitrifiers were known to inhibit methanogen growth. Experimental results showed consistent higher $\mathrm{H}_{2}$ yield in inoculated reactor compared to control reactor, where in the second cycle $\mathrm{H}_{2}$ production increased $100 \%$ compared to the control.
\end{abstract}

\section{Introduction}

Fossil fuel usage brought more harm than good. Negative environmental consequences such as climate change and the rising global temperature level are just some to name. Clean, renewable energy must take the stage as the world's leading energy source in the near future to minimize the irreversible impacts caused by centuries-long pollution to our atmosphere.

Hydrogen gas or $\mathrm{H}_{2}$ for short, is one of the most promising leading candidates of renewable energy of the future. It emits almost zero carbon dioxide [1] and nitrous oxide [2] to the atmosphere, has high calorific value at $120-140 \mathrm{MJ} / \mathrm{kg}$ compared to petrol at $44-46$ $\mathrm{MJ} / \mathrm{Kg}$, and it is not perceived negatively in the politic and economic sector [3].

Microbial Electrolysis Cell (MEC for short) system is one of the alternative method for $\mathrm{H}_{2}$ production that has been gaining traction due to its relatively cheap and easy to obtain substrate (e.g. wastewater) that has multiple possible applications, ranging from energy production $\left(\mathrm{H}_{2}\right.$ and $\left.\mathrm{CH}_{4}\right)$ to wastewater treatment [4] [5].

A common problem that hinders potential application of MEC usage to produce $\mathrm{H}_{2}$ is the presence of methanogenic bacteria in the system that produces $\mathrm{CH}_{4}$ out of the produced $\mathrm{H}_{2}$ [5] [6]. This reduces the possible yield of $\mathrm{H}_{2}$, and this is very apparent in a membrane-less reactor [6]. Although this problem can usually be minimalized by using an ion exchange membrane to separate the cathodic and anodic chamber, such usage of membrane hinder scale-up application as the membrane consists a huge portion of MEC manufacturing cost [7] and must be routinely replaced, making MEC technology expensive.

One of the method that has yet to be tried in order to tackle the presence of methanogens is by biological competition. Denitrifier is a class of bacteria that are capable of reducing nitrates present in wastewater [8] to a reduced form (e.g. nitrite, nitrous oxide) that will ultimately result in $\mathrm{N}_{2}$ gas, in which the process is accelerated in an anaerobic environment [9]. Research showed that in a contained system, metabolites produced by denitrifier is known to inhibit methanogenic activity [10] [11] [12].

Our team aimed to validate whether or not the presence of denitrifiers in a MEC system will assist with $\mathrm{H}_{2}$ production by inhibiting the methanogens. This method is relatively cheap and environmentally-friendly as denitrifier is well-known to be employed in bioremediation efforts [8]. The chosen denitrifier is the Pseudomonas stutzeri because aside from the relatively easy access to the culture, it is known to be able to perform complete denitrification pathway [13] and is able to grow in an anaerobic condition.

The MEC system that we used is made of $20-\mathrm{ml}$ glass vial with a working volume of $15 \mathrm{ml}$. The reactor will be membrane-less so as to reduce cost [14] for potential scale-up. The wastewater sludge is obtained

Corresponding author: nana@che.ui.ac.id 
from a local wastewater reservoir located in Jakarta. Denitrifier culture of $P$. stutzeri is obtained from InaCC LIPI.

\section{Methods}

\subsection{MEC Reactor Design}

We based our reactor design on a study by Call \& Logan (2011) [15] in which a small-scale, affordable reactor was assembled using commercially-available materials. Based on the picture below, we used a single-chambered, membraneless reactor made of $20 \mathrm{ml}$ clear glass vials (Agilent) crimped with appropriate rubber septum (E) used to prevent gas exchange. Anodes (A) used were isomolded graphite plates with a thickness of 0.125 inches (Grade GM-10; GraphiteStore.com Inc.) cut manually to an average dimension of $1.5-\mathrm{cm} \times 1.0-\mathrm{cm}(\mathrm{L}$ $\mathrm{x}$ W). Cathodes (B) used were stainless steel mesh cut to the same dimension of its respective anode pair. In order to establish electrical connection with the electrodes, titanium wire (0.08-cm diameter, Grade 2; Ti-Shop.com) (C) and stainless steel wire $(0.08-\mathrm{cm}$ diameter) (D) are used for the anode and cathode respectively. All electrodes and wires and polished beforehand with sandpaper, sonicated for 20-min, and rinsed three times by Type-1 water. Only for anode, after sonication it is soaked overnight in $1 \mathrm{~N} \mathrm{HCl}$ solution.

In order to connect the wire with the electrodes, a hand drill $(0.08-\mathrm{cm}$ diameter drill tip) is used to bore 2 holes on the electrodes. The wire is then inserted into the first hole, then bent at the end to insert it at the second hole. The wire is then crimped to the electrode by a plier in order to create a tight connection. Internal resistances for the electrodes will then be tested with a multimeter. Only electrodes with resistances below $2 \Omega$ will be used in the experiment. The wire side of the electrode will then be inserted through the rubber septum, and then crimped with the glass vial to create a finished reactor. The electrode spacing is kept at a minimum distance of $0.5-\mathrm{cm}$. The total working volume is $15 \mathrm{ml}$, providing headspace for gas to accumulate.

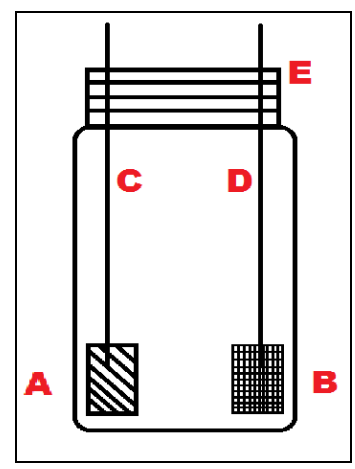

Fig 1. MEC Reactor Design

\subsection{Anaerobic Procedure}

After crimp sealing the reactor with rubber septum, the reactor will then be vacuumed and flushed for three cycles with Ultra-High Purity $\mathrm{N}_{2}$ gas to purge oxygen out of the system, creating an anaerobic environment. Flushing and vacuuming is carried out by means of tube with syringe attached to the end, connected to each respective sources. For flushing then the tube is attached to a $\mathrm{N}_{2}$ source and for vacuuming it is attached to a vacuum pump. Each time flushing is about to be carried, the syringe will pierce the rubber septum to minimize gas exchange between reactors and the outside environment.

The flushing cycles are as follows: firstly vial is pumped with $\mathrm{N}_{2}$ for $10 \mathrm{~s}$, then the vacuum line is added to simultaneously pump $\mathrm{N}_{2}$ and vacuum the air for $20 \mathrm{~s}$, lastly the $\mathrm{N}_{2}$ line is removed and the vial is vacuumed empty again for $10 \mathrm{~s}$. Whenever $\mathrm{N}_{2}$ is flushed, the vial will be tilted so as to submerge the needle tip with the liquid inside in order to flush aqueous oxygen out of the liquid.

After three cycles of flushing and vacuuming, the reactor will then be filled again with $\mathrm{N}_{2}$ for 5-7 s to build positive pressure inside the system to prevent outside air from being sucked inside the reactor. All reactors will then be autoclaved to sterilize them. The picture below illustrates the flushing and vacuuming process. Note the formed bubbles that indicates gas exchange between liquid and gas phase.

\subsection{MEC Reactor Operation}

A programmable power supply is used to supply energy to the system in order to drive the reaction that produces $\mathrm{H}_{2}$ in the cathode, the required potential being $0.7 \mathrm{~V}$ Call \& Logan (2011) [15]. The output is made parallel by using two sets of 4 test leads soldered together at one end, then attached to the positive and negative terminal of the power supply. This is done in order to supply power simultaneously to more than one reactors. The supplied current is set at $1 \mathrm{~A}$.

Each test leads will then be connected to the reactors using alligator clips, with the anode connected to the positive terminal and the cathode connected to the negative terminal. Between the power supply and the anode, a $10 \Omega$ resistor was used in order to record the voltage of the reactor. A multimeter (model $109 \mathrm{~N}$; APPA Technology Corp.) was used to record the voltage in every 10 -s interval and was connected to a computer to visualize the graph.

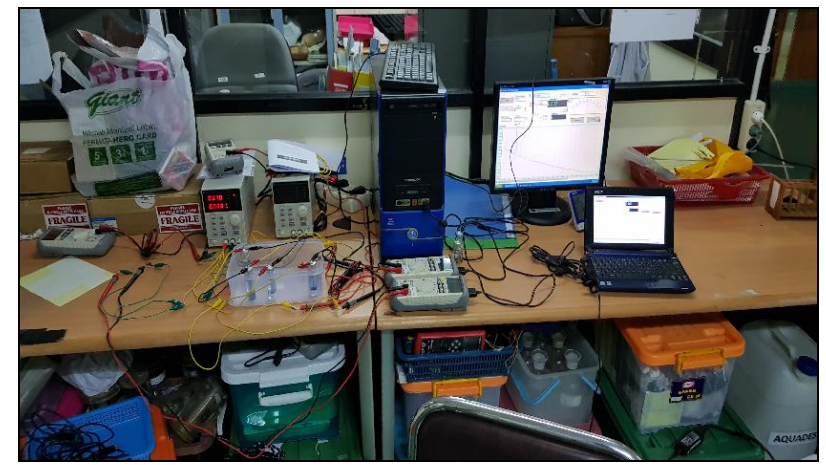

Fig 2. MEC reactor setup 
The medium used was formulated according to Tugtas (2007) [16] but without the vitamin stock and kept in sterile, anaerobic glass vial (Agilent) that had been crimped with rubber septum. The procedures for anaerobic flushing and sterilization are the same akin to that for the reactor. As the medium contains $0.0002 \%$ $\mathrm{w} / \mathrm{v}$ methylene blue in its formulation, it gives off a light blue coloration that will change to colorless in an environment where oxygen has been depleted [17]. Medium is replaced after each gas chromatography analysis by using sterile, disposable syringe inside a laminar hood to minimize risk of contamination in which depleted medium is sucked with a syringe, then the new batch of medium is added with a new syringe. Each replacement of medium adds approximately same amount of volume as the working volume, which is 15 $\mathrm{ml}$.

The experiment will consist of three reactors running in parallel, with each reactor being given the same treatment (ex: medium replacement, current input, etc.). The first reactor will consists of wastewater sludge, medium, and inoculum of denitrifier P. stutzeri (B1205, InaCC LIPI). The second reactor functions as a negative control, containing only wastewater sludge and medium. The third reactor also functions as a negative control, containing only the medium. Inoculum of $P$. stutzeri were grown beforehand in the same medium inside a sterile, anaerobic glass vial before being transferred with a syringe to the first reactor in a laminar hood.

Wastewater sludge was obtained from Waduk Ria Rio (Pulo Mas, Jakarta) to serve as the source of mixed exoelectrogenic culture and stored beforehand in a falcon tub inside a refrigerator. Wastewater will be diluted a hundredfold before being used in the experiment, in which phosphate-buffered saline was used as the medium of dilution. The first reactor contains $1.5 \mathrm{ml}$ diluted wastewater sludge, $1.5 \mathrm{ml}$ inoculum, and $12 \mathrm{ml}$ medium. The second reactor contains $1.5 \mathrm{ml}$ wastewater sludge and $13.5 \mathrm{ml}$ medium. The third reactor only contains $15 \mathrm{ml}$ medium. From hereafter, the first reactor shall be labelled "A", the second reactor labelled "B", and the third reactor labelled " $\mathrm{C}$ ".

\subsection{Analysis}

In this study, accumulated gas in the headspace will be analysed after certain intervals using a gas chromatograph. This is due to the fact that if accumulated gas is left too long, $\mathrm{H}_{2}$ may get fully converted into methane by methanogens. The gas chromatograph used (model 8A, Shimadzu) is equipped with TCD (thermal conductivity detector) and utilizing argon as the carrier gas in order to detect $\mathrm{H}_{2}$. In each analysis, $1 \mathrm{ml}$ of headspace gas was taken with chromatography syringe to ensure no gas escapes from the syringe, and then injected into the gas chromatograph. From the resulting chromatogram, composition of the gas can be deduced and quantified. The section below will detail the result of each cycle of reactor operation.

\section{Result and Discussion}

\subsection{Visual Observation}

Visual observation is done for the first two cycles in order to verify the operation of the reactors. The observed elements were the medium coloration and the presence of gas bubbles in the cathode. The color of the medium will change from light blue to colorless in an oxygen-free environment, which indicates growth of microorganism that eats the aqueous oxygen [17]. Cathodic bubbles were observed as it is an indicator of evolution of $\mathrm{H}_{2}$ gas. The produced $\mathrm{H}_{2}$ will bubble due to its low solubility in water [18] and rise up to accumulate in the reactor headspace.

Observation of the first cycle (after \pm 22 hrs elapsed of reactor operation) showed that only reactor A and $\mathrm{B}$ got discoloured in their medium, with reactor $\mathrm{C}$ retaining its light blue methylene blue coloration and reactor $\mathrm{A}$ being more colorless than $\mathrm{B}$. This proves that there was growth of microorganism in reactors inoculated with inoculum and wastewater, but not in the reactor containing only medium. It also indirectly proves that the sterilization process for the medium was successful. Furthermore, bubbles of gas were found attached on the cathode of reactor A and B, meaning that the MEC setup was successful and that hydrogen gas is being produced. On the contrary, no bubbles were observed in reactor $\mathrm{C}$ which indicates that the voltage used was high enough to enable $\mathrm{H}_{2}$ production by MEC mechanism but low enough that $\mathrm{H}_{2}$ is not produced by any other sort of mechanism such as water electrolysis [15].

Observation of the second cycle (after $\pm 20 \mathrm{hrs}$ elapsed of reactor operation) showed that reactor A and $\mathrm{B}$ had gotten completely colorless, with reactor C retaining its coloration as predicted. Compared to the observation on the first cycle at around the same interval ( $\pm 22 \mathrm{hrs}$ ), the rate of discoloration was much faster. It is presumed that the microorganism had acclimatized to the environment of the reactor and thus reduced the methylene blue at a much faster rate.

\subsection{Chromatographic Analysis}

\section{First Cycle}

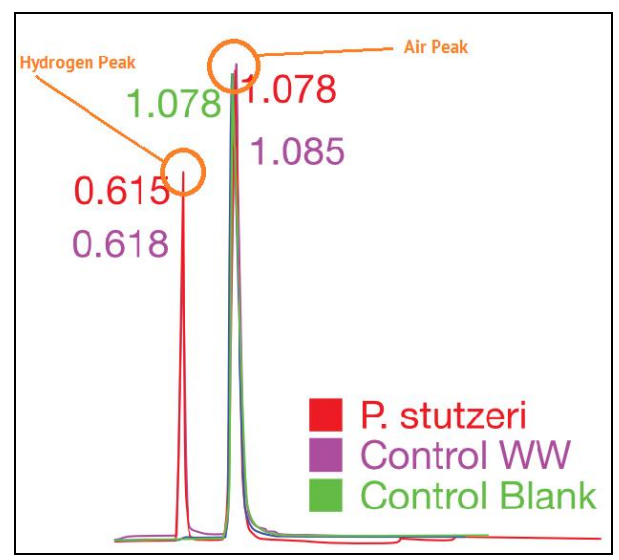

Fig 3. Chromatogram for the first cycle (69 hours) 
Analysis of the headspace taken after $69 \mathrm{hrs}$ of reactor operation is shown in Fig. 3 above. Chromatogram result for reactor $\mathrm{C}$ yielded no peak of hydrogen. This shows that indeed at the applied voltage of $0.7 \mathrm{~V}$, no $\mathrm{H}_{2}$ was produced by other mechanism (such as water electrolysis) other than that of MEC working principle, meaning that the applied voltage was appropriate in this study. On the other hand, both reactor $\mathrm{A}$ and $\mathrm{B}$ showed peak for hydrogen at around 0.6 , with reactor A's headspace composed of $29.06 \% \mathrm{H}_{2}$ and reactor B's headspace composed of $24.63 \% \mathrm{H}_{2}$. This proved that aside from the fact that the sludge used contained living exoelectrogen culture, $\mathrm{H}_{2}$ was also produced meaning that the set-up of the experiment was successful. The small fluctuations seen on the chromatogram is presumed to be noise and thus not analysed. However, the predicted production difference was not yet seen in this cycle, which may be caused by either long reactor operation time that made $\mathrm{H}_{2}$ evolution rate at a slower interval ot observed or simply that the methanogens have yet to be active in the reactor. The result of the first cycle is summarized in Figure 4 below. From hereafter, chromatogram result will not be displayed as the retention time of the peaks for the consequent analysis remain the same.

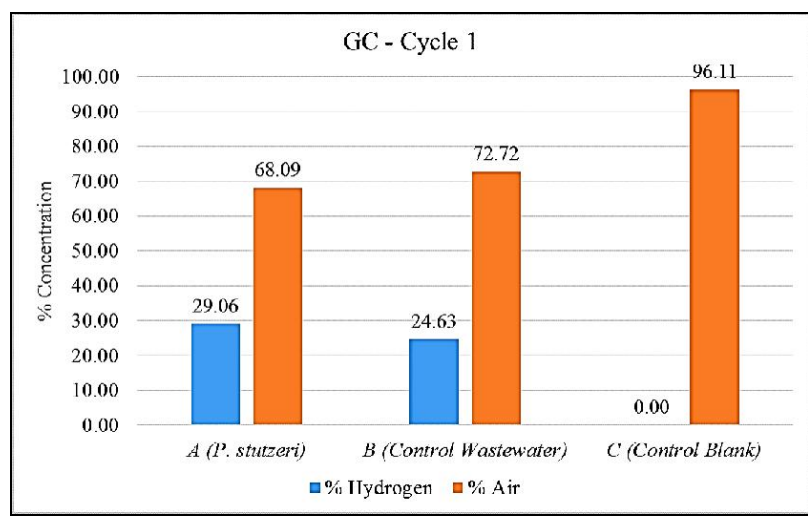

Fig 4. GC result for the first cycle (69 hours)

\section{Second Cycle}

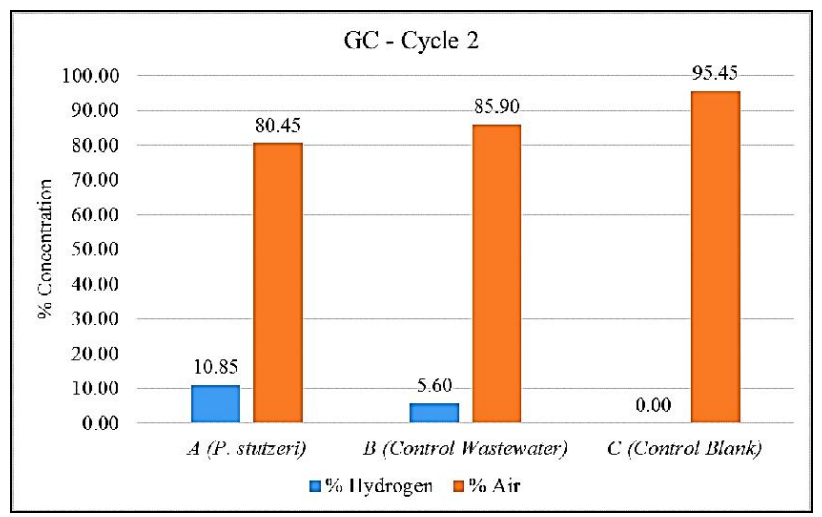

Fig 5. GC result for the second cycle (39 hours)

Analysis of the headspace taken after $39 \mathrm{hrs}$ of reactor operation is shown in Fig. 5 above. Chromatogram result for reactor $\mathrm{C}$ still did not yield any peak of hydrogen as predicted. As consequent analysis proved the premise that the voltage applied was suitable, for subsequent cycle we did not analyse for reactor $\mathrm{C}$ anymore. On the other hand, both reactor $\mathrm{A}$ and $\mathrm{B}$ showed peak for hydrogen at around 0.6 , with reactor A's headspace composed of $10.85 \% \mathrm{H}_{2}$ and reactor B's headspace composed of $5.60 \% \mathrm{H}_{2}$.

This result proved the hypothesis in which the presence of the denitrifer inside the reactor is beneficial, in which in this case the $\mathrm{H}_{2}$ production is increased by $100 \%$. Although the result showed higher production in reactor inoculated with denitrifier, due to the scope of this research, the direct mechanism behind these phenomena is yet to be verified, aside from the possibility that products of the denitrifier metabolism inhibited methanogenic activites [10] [11] [12] and thus reduced methanogen growth, increasing the net $\mathrm{H}_{2}$ harvested in the reactor.

Another thing to note is that the faster analysis time (39 hrs vs. $69 \mathrm{hrs}$.) allowed better insight as to which reactor can produce higher yield of $\mathrm{H}_{2}$ in a shorter amount of time, something important if $\mathrm{H}_{2}$ production by means of MEC is to be scaled-up.

\section{Third Cycle}

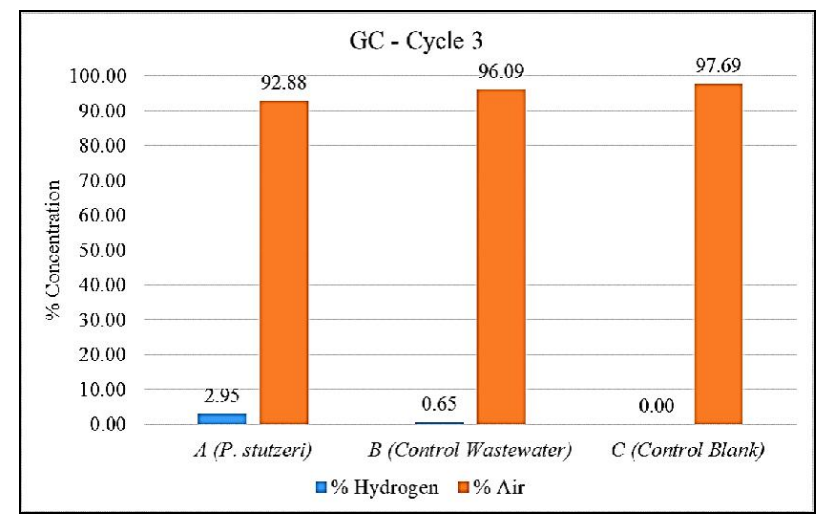

Fig 6. GC result for the third cycle (46 hours)

Analysis of the headspace taken after $46 \mathrm{hrs}$ of reactor operation is shown in Fig. 6 above. Only reactor A showed peak for hydrogen at around 0.6, with reactor A's headspace composed of $2.95 \% \mathrm{H}_{2} . \mathrm{H}_{2}$ peak was not observed with reactor $\mathrm{B}$. It is presumed that even after longer operational time compared to the previous cycle (46 hrs vs. $39 \mathrm{hrs}$ ) the $\mathrm{H}_{2}$ production rate has slowed down or even not produced at all. This could mean that either the denitrifiers had entered the lag phase due to absence of nutrient or the exoelectrogenic culture from the wastewater sludge which had entered lag phase.

\section{Fourth Cycle}




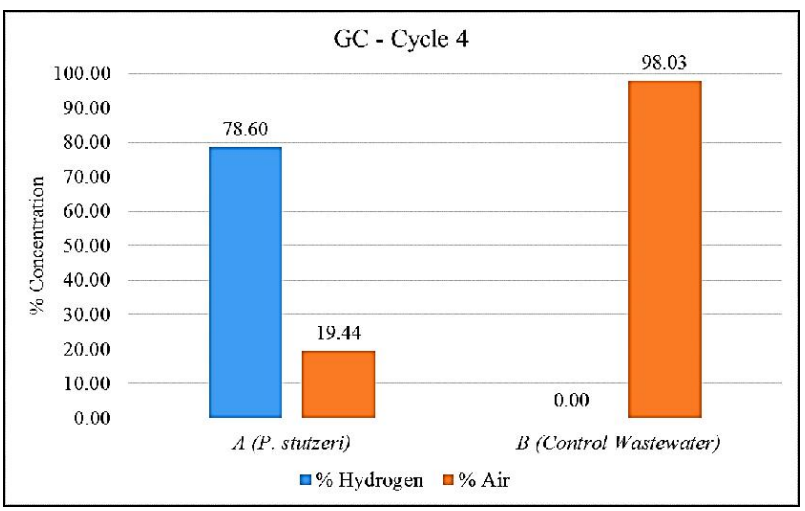

Fig 7. GC result for the fourth cycle (71 hours)

Analysis of the headspace taken after $71 \mathrm{hrs}$ of reactor operation is shown in Fig. 7 above. Although reactor $\mathrm{B}$ consistently yielded no peak for $\mathrm{H}_{2}$ akin to the previous cycle, hydrogen peak was observed yet again in reactor A. The headspace analysis for reactor A showed that the $\mathrm{H}_{2}$ composition was $78.60 \%$, the highest out of any cycle. It is presumed that although the $\mathrm{H}_{2}$ production rate slowed down in the previous cycle that may lead to either denitrifier/exoelectrogen entering lag phase, not all died. This is proven that by supplying fresh batch of medium on a smaller number of microorganism (some must be dead on the lag phase), allowed the microorganism to produce $\mathrm{H}_{2}$ again by means of the MEC. However, this large jump in $\mathrm{H}_{2}$ production needs to be analysed further to exactly determine the real cause of this huge leap of $\mathrm{H}_{2}$ generated. The overall result of the experiment is summarized in Figure 8 below.

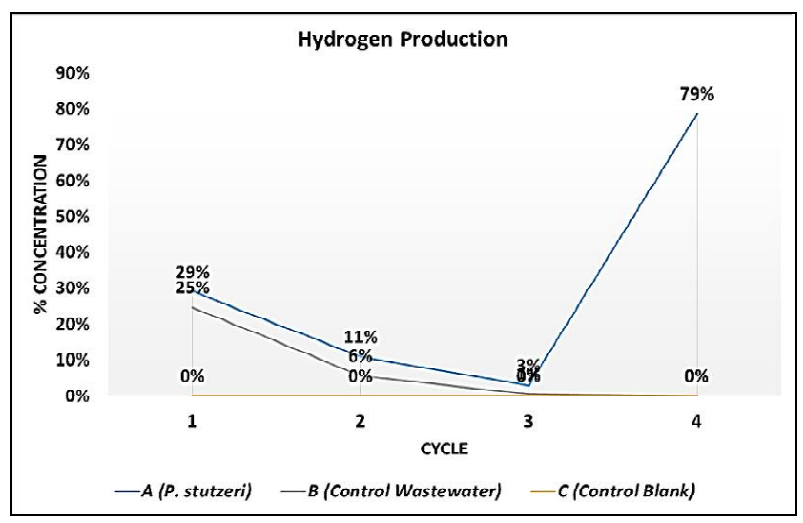

Fig 8. Overall Composition of $\mathrm{H}_{2}$ on Reactor Headspace

Something to note is that, given that all reactors were given the same kind of treatment, the same amount and type of food, the same source of wastewater sludge, and the same interval of headspace analysis, only reactor A that has been inoculated with denitrifier $P$. stutzeri was capable of maintaining its $\mathrm{H}_{2}$ production on subsequent cycle and even saw an increase in production, while $\mathrm{H}_{2}$ production by reactor $\mathrm{B}$ saw a decline between the first and second cycle. We posit that the presence of denitrifier in a MEC reactor is highly beneficial if one wishes to produce $\mathrm{H}_{2}$ by means of MEC as it increases $\mathrm{H}_{2}$ production yield. Further research may be required in order to truly assess the interaction between the three types of organism present in the reactor (denitrifier, exoelectrogen, and methanogen) as well as its validity in a scale-up reactor.

\section{Conclusion}

The conclusion obtained from this research are that by using small-scale MEC reactor to produce $\mathrm{H}_{2}$ as the clean, renewable fuel of the future, it is beneficial to introduce denitrifier microorganism in order to tackle the methanogenesis activity present in wastewater that consumes the produced $\mathrm{H}_{2}$. A reactor inoculated with $P$. stutzeri running in parallel with an uninoculated one showed higher $\mathrm{H}_{2}$ yield and better sustainability of $\mathrm{H}_{2}$ production, in which in the second cyle $\mathrm{H}_{2}$ production increased $100 \%$ compared to control reactor. Further research is still needed, however, in order to assess the truly assess the interaction between the two microorganism and the mechanism that leads to increase of $\mathrm{H}_{2}$ evolution, as well as explaining the high jump in $\mathrm{H}_{2}$ production in the last cycle.

\section{References}

[1] A. Kadier, M.S. Kalil, P. Abdeshahian, K. Chandrasekhar, A. Mohamed, N.F. Azman, W. Logroño, Y. Simayi, A.A. Hamid, Renew. Sustain. Energy Rev. 61 (2016) 501-525.

[2] P. Hoffmann, U.K. Mirza, Electron. Green J. (2002).

[3] P. Kumar, R. Britter, N. Gupta, J. Fuel Cell Sci. Technol. 6 (2009) 021009.

[4] H. Liu, S. Grot, B.E. Logan, Environ. Sci. Technol. 39 (2005) 4317-4320.

[5] T.H.J.A. Sleutels, A. Ter Heijne, C.J.N. Buisman, H.V.M. Hamelers, ChemSusChem 5 (2012) 1012-1019.

[6] L. Lu, N. Ren, X. Zhao, H. Wang, D. Wu, D. Xing, Energy Environ. Sci. 4 (2011) 1329.

[7] R.A. Rozendal, H.V.M. Hamelers, K. Rabaey, J. Keller, C.J.N. Buisman, Trends Biotechnol. 26 (2008) 450-459.

[8] D. Clifford, X. Liu, J. Am. Water Works Assoc. (1993) 135-143.

[9] W. Zumft, Microbiol Mol Biol Rev 61 (1997) 533-616.

[10] K.C. Chen, Y.F. Lin, Water Res. 27 (1993) 1749-1759.

[11] A.E. Tugtas, S.G. Pavlostathis, Biotechnol. Bioeng. 96 (2007) 444-455.

[12] A.E. Tugtas, U. Tezel, S.G. Pavlostathis, Biotechnol. Bioeng. 105 (2010) 98-108.

[13] C.A. Carlson, J.L. Ingraham, Appl. Environ. Microbiol. 45 (1983) 1247-1253.

[14] L. Rago, Y. Ruiz, J.A. Baeza, A. Guisasola, P Cortés, Bioelectrochemistry 106 (2015) 359368.

[15] D.F. Call, B.E. Logan, Biosens. Bioelectron. 26 (2011) 4526-4531.

[16] A.E. Tugtas, (2007).

[17] D. Karakashev, D. Galabova, I. Simeonov, World J. Microbiol. Biotechnol. 19 (2002) 233- 
238.

[18] A. Kadier, Y. Simayi, P. Abdeshahian, N. Farhana, K. Chandrasekhar, M. Sahaid, ALEXANDRIA Eng. J. (2015). 